\title{
APPLICATION OF THE VARIATIONAL ITERATION METHOD FOR SYSTEM OF NONLINEAR VOLTERRA'S INTEGRO-DIFFERENTIAL EQUATIONS
}

\author{
Saeid Abbasbandy ${ }^{1}$, Elyas Shivanian ${ }^{2}$ \\ Department of Mathematics, Imam Khomeini International \\ University, Ghazvin, 34149-16818, Iran \\ 1abbasbandy@yahoo.com \\ ²eshivanian@gmail.com
}

\begin{abstract}
In this paper, the variational iteration method is proposed to solve system of nonlinear Volterra's integro-differential equations. Four numerical examples are illustrated by this method. The results reveal that this method is very effective and highly promising in comparison with other numerical methods.
\end{abstract}

Key Words- Variational iteration method, System of nonlinear Volterra's integrodifferential equations

\section{INTRODUCTION}

The variational iteration method $[1,2]$, which is a modified general Lagrange multiplier method [3] has been shown to solve effectively, easily and accurately, a large class of nonlinear problems with approximations which converge quickly to accurate solutions. It was successfully applied to autonomous ordinary differential equation [4], to nonlinear partial differential equations with variable coefficients [5], to Schrödinger$\mathrm{KDV}$, generalized KDV and shallow water equations [6], to Burgers' and coupled Burgers' equations [7], to the linear Helmoltz partial differential equation [8] and recently to nonlinear fractional differential equations with Caputo differential derivative [9], and other fields [10-12, 28-31]. On the other hand, one of the interesting topics among researchers is solving integro-differential equations. In fact, integro-differential equations arise in many physical processes, such as glass-forming process [13], nanohydrodynamics [14], drop wise condensation [15], and wind ripple in the desert [16]. There are various numerical and analytical methods to solve such problems, for example, the homotopy perturbation method [17], the Adomian decomposition method [18], but each method limits to a special class of integro-differential equations. J.H. He used the variational iteration method for solving some integro-differential equations [19]. This Chinese mathematician chooses [19] initial approximate solution in the form of exact solution with unknown constants. M. Ghasemi et al solved the nonlinear Volterra's integro-differential equations [26] by using homotopy perturbation method. In [21], the variational iteration method was applied to solve the system of linear integro-differential equations. Also, J. Biazar et al solved systems of integro-differential equations by He's homotopy perturbation method [22]. 
The purpose of this paper is to extend the analysis of the variational iteration method to solve the system of general nonlinear Volterra's integro-differential equations which is as follows:

$$
\begin{aligned}
u_{1}^{(m)}(x)= & H_{1}\left(x, u_{2}(x), \ldots, u_{2}^{(m)}(x), u_{3}(x), \ldots, u_{3}^{(m)}(x), \ldots, u_{n}(x), \ldots, u_{n}^{(m)}(x)\right) \\
& +\int_{0}^{x} \mathrm{~K}_{1}\left(x, t, u_{1}(t), \ldots, u_{1}^{(m)}(t), \ldots, u_{n}(t), \ldots, u_{n}^{(m)}(t)\right) \mathrm{d} t, \\
u_{2}^{(m)}(x)= & H_{2}\left(x, u_{1}(x), \ldots, u_{1}^{(m)}(x), u_{3}(x), \ldots, u_{3}^{(m)}(x), \ldots, u_{n}(x), \ldots, u_{n}^{(m)}(x)\right) \\
& +\int_{0}^{x} \mathrm{~K}_{2}\left(x, t, u_{1}(t), \ldots, u_{1}^{(m)}(t), \ldots, u_{n}(t), \ldots, u_{n}^{(m)}(t)\right) \mathrm{d} t, \\
& \cdot \\
& \cdot \\
& \cdot \\
u_{n}^{(m)}(x)= & H_{n}\left(x, u_{1}(x), \ldots, u_{1}^{(m)}(x), u_{2}(x), \ldots, u_{2}^{(m)}(x), \ldots, u_{n-1}(x), \ldots, u_{n-1}^{(m)}(x)\right) \\
& +\int_{0}^{x} \mathrm{~K}_{\mathrm{n}}\left(x, t, u_{1}(t), \ldots, u_{1}^{(m)}(t), \ldots, u_{n}(t), \ldots, u_{n}^{(m)}(t)\right) \mathrm{d} t,
\end{aligned}
$$

In system (1), $m$ is order of derivatives and the continuous several variables functions $H_{i}$ and $K_{i}, i=1,2, \ldots, n$ are given, the solutions to be determined are $u_{i}(x)$, $i=1,2, \ldots, n$. In Section 2 , the basic ideas of variational iteration method are stated. Three examples are given in Section 3 also; we compare our results with another numerical method in this section. It is shown that this method is very simple and effective. Finally conclusions are stated in Section 4.

\section{BASIC IDEAS OF VARIATIONAL ITERATION METHOD}

To illustrate the basic concept of variational iteration method, we consider the following general nonlinear system

$$
L[u(x)]+N[u(x)]=\psi(x),
$$

where $L$ is a linear operator, $N$ is a nonlinear operator and $\psi(x)$ is a given continuous function. According to the variational iteration method [10, 23-25], we can construct a correction functional in the form

$$
u_{k+1}(x)=u_{k}(x)+\int_{0}^{x} \lambda(s)\left[L\left[u_{k}(s)\right]+N\left[\tilde{u}_{k}(s)\right]-\psi(x)\right] \mathrm{d} s
$$

where $u_{0}(x)$ is an initial approximation with possible unknowns, $\lambda$ is a Lagrange multiplier which can be identified optimally via variational theory, the subscript $k$ denotes the $k$ th approximation, and $\tilde{u}_{k}$ is considered as a restricted variation [10, 23], i.e. $\delta \tilde{u}_{k}=0$. It is shown this method is very effective and easy for linear problem, its 
exact solution can be obtained by only one iteration, because $\lambda$ can be exactly identified.

For solving (1) by the variational iteration method, for simplicity, we consider all of terms as restricted variation except $u_{i}^{m}(x), i=1,2, \ldots, n$. According to the variational iteration method, we derive correction functional as follow:

$$
\begin{aligned}
u_{i, k+1}(x)=u_{i, k}(x)+\int_{0}^{x} & \lambda_{i}(s)\left[u_{i, k}^{(m)}(s)-H_{i}\left(s, \tilde{u}_{1, k}(s), \ldots, \tilde{u}_{1, k}^{(m)}(s)\right), \ldots,\right. \\
& \left.\left.\tilde{u}_{i-1, k}(s), \ldots, \tilde{u}_{i-1, k}^{(m)}(s)\right), \tilde{u}_{i+1, k}(s), \ldots, \tilde{u}_{i+1, k}^{(m)}(s)\right), \\
& \left.\tilde{u}_{n, k}(s), \ldots, \tilde{u}_{n, k}^{(m)}(s)\right)-\int_{0}^{s} K_{i}\left(s, t, \tilde{u}_{1, k}(t), \ldots,\right. \\
& \left.\left.\tilde{u}_{1, k}^{(m)}(t), \ldots, \tilde{u}_{n, k}(t), \ldots, \tilde{u}_{n, k}^{(m)}(t)\right) \mathrm{d} t\right] \mathrm{ds}
\end{aligned}
$$

for $i=1,2, \ldots, n$ and the stationary conditions of the above correction functional can be expressed as follows:

$$
\begin{aligned}
& \lambda_{i}^{(m)}(s)=0 \\
& 1+\left.(-1)^{m-1} \lambda_{i}^{(m-1)}(s)\right|_{s=x}=0, \\
& \left.\lambda_{i}^{(j)}(s)\right|_{s=x}=0, \quad j=1,2, \ldots, m-2, \quad i=1,2, \ldots, n \\
& \left.\lambda_{i}(s)\right|_{s=x}=0 .
\end{aligned}
$$

The Lagrange multiplier, therefore, can be identified as follows:

$$
\lambda_{i}(s)=\frac{(-1)^{m}}{(m-1) !}(s-x)^{m-1}
$$

As a result, we obtain the following iteration formulas

$$
\begin{aligned}
u_{i, k+1}(x)=u_{i, k}(x)+\int_{0}^{x} & \frac{(-1)^{m}}{(m-1) !}(s-x)^{m-1}\left[u_{i, k}^{(m)}(s)-H_{i}\left(s, u_{1, k}(s), \ldots, u_{1, k}^{(m)}(s)\right), \ldots,\right. \\
& \left.\left.u_{i-1, k}(s), \ldots, u_{i-1, k}^{(m)}(s)\right), u_{i+1, k}(s), \ldots, u_{i+1, k}^{(m)}(s)\right), \\
& \left.\ldots, u_{n, k}(s), \ldots, u_{n, k}^{(m)}(s)\right)-\int_{0}^{s} K_{i}\left(s, t, u_{1, k}(t), \ldots,\right. \\
& \left.\left.\tilde{u}_{1, k}^{(m)}(t), \ldots, \tilde{u}_{n, k}(t), \ldots, \tilde{u}_{n, k}^{(m)}(t)\right) \mathrm{d} t\right] \mathrm{ds}, \quad i=1,2,3, \ldots, n .
\end{aligned}
$$

\section{APPLICATIONS}

In this section, we present some examples to show efficiency and high accuracy of the variational iteration method for solving system of nonlinear Volterra's integrodifferential equations (1). 
Example 3.1: [22] Let us first consider the system of nonlinear integro-differential equations as follow:

$$
\begin{aligned}
& u^{\prime \prime}(x)=1-\frac{1}{3} x^{3}-\frac{1}{2} v^{\prime 2}(x)+\frac{1}{2} \int_{0}^{x}\left(u^{2}(t)+v^{2}(t)\right) \mathrm{d} t, \\
& v^{\prime \prime}(x)=-1+x^{2}-x u(x)+\frac{1}{4} \int_{0}^{x}\left(u^{2}(t)-v^{2}(t)\right) \mathrm{d} t, \\
& u(0)=1, \quad u^{\prime}(0)=2, \quad v(0)=-1, \quad v^{\prime}(0)=0,
\end{aligned}
$$

with the exact solutions

$$
u(x)=x+e^{x}, \quad v(x)=x-e^{x} .
$$

According to (2) we have the following iteration formulas

$$
\begin{aligned}
& u_{k+1}(x)=u_{k}(x)+\int_{0}^{\mathrm{x}}(s-x)\left[u_{k}^{\prime \prime}(s)-1+\frac{1}{3} s^{3}+\frac{1}{2} v_{k}^{\prime 2}(s)-\frac{1}{2} \int_{0}^{\mathrm{s}}\left(u_{k}^{2}(t)+v_{k}^{2}(t)\right) \mathrm{d} t\right] \mathrm{d} s, \\
& v_{k+1}(x)=v_{k}(x)+\int_{0}^{\mathrm{x}}(s-x)\left[v_{k}^{\prime \prime}(s)+1-s^{2}+s u_{k}(s)-\frac{1}{4} \int_{0}^{\mathrm{s}}\left(u_{k}^{2}(t)-v_{k}^{2}(t)\right) \mathrm{d} t\right] \mathrm{d} s .
\end{aligned}
$$

Now, we choose initial approximations $u_{0}(x)=2 x+1$ and $v_{0}(x)=1$ which satisfy initial conditions then, we obtain

$$
\begin{aligned}
u_{1}(x)= & 1+2 x+\frac{1}{2} x^{2}+\frac{1}{6} x^{3}+\frac{1}{12} x^{4}+\frac{1}{60} x^{5} \\
v_{1}(x)= & -1-\frac{1}{2} x^{2}-\frac{1}{6} x^{3}-\frac{1}{24} x^{4}+\frac{1}{60} x^{5} \\
u_{2}(x)= & 1+2 x+\frac{1}{2} x^{2}+\frac{1}{6} x^{3}+\frac{1}{24} x^{4}+\frac{1}{120} x^{5}+\frac{1}{720} x^{6}+\frac{17}{5040} x^{7}+\frac{1}{672} x^{8}+\frac{53}{120960} x^{9} \\
& -\frac{1}{103680} x^{10}+\frac{1}{228096} x^{11}+\frac{1}{1900800} x^{12}+\frac{1}{6177600} x^{13}, \\
v_{2}(x)= & -1-\frac{1}{2} x^{2}-\frac{1}{6} x^{3}-\frac{1}{24} x^{4}-\frac{1}{120} x^{5}-\frac{1}{720} x^{6}-\frac{11}{10080} x^{7}+\frac{13}{241920} x^{9} \\
& +\frac{17}{1036800} x^{10}+\frac{47}{11404800} x^{11}+\frac{1}{1267200} x^{12},
\end{aligned}
$$

It is obvious that the iterations converge to exact solutions. This example has been solved by the homotopy perturbation method in [22]. In order to show the efficiency and high accuracy of the present method, in Figure (1), we plotted the error functions, i.e. 


$$
\begin{aligned}
& E^{\text {vim }}(x)=y_{\text {exact }}(x)-y_{\text {vim }}(x), \\
& E^{\text {hom }}(x)=y_{\text {exact }}(x)-y_{\text {hom }}(x) .
\end{aligned}
$$

As we can see, this method is very effective and it is applied very convenient. Also, the obtained solutions with 6 iterations are showed in Figure (2) graphically.

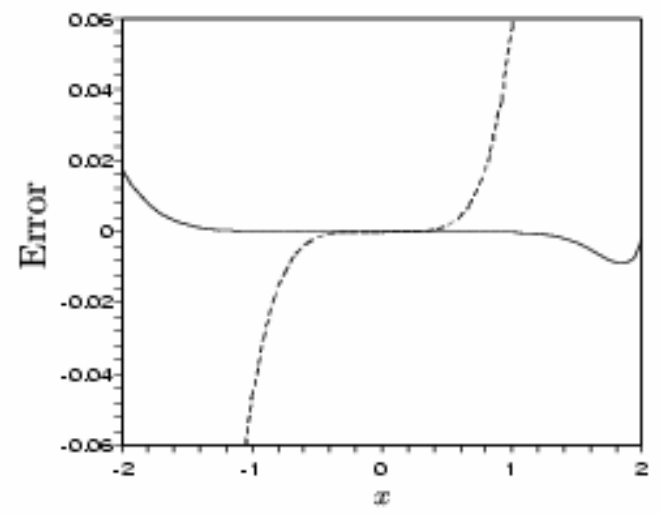

(a)

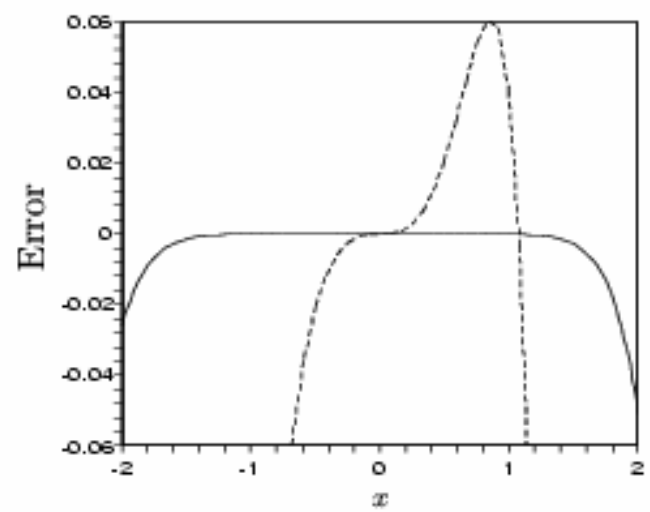

(b)

Fig. 1: (a): Solid line: The error of VIM with third iteration for $u(x)$; dashed line: The error of homotopy method with 3 terms for $u(x)$; (b): Solid line: The error of VIM with third iteration for $v(x)$; dashed line: The error of homotopy method with 3 terms for $v(x)$.

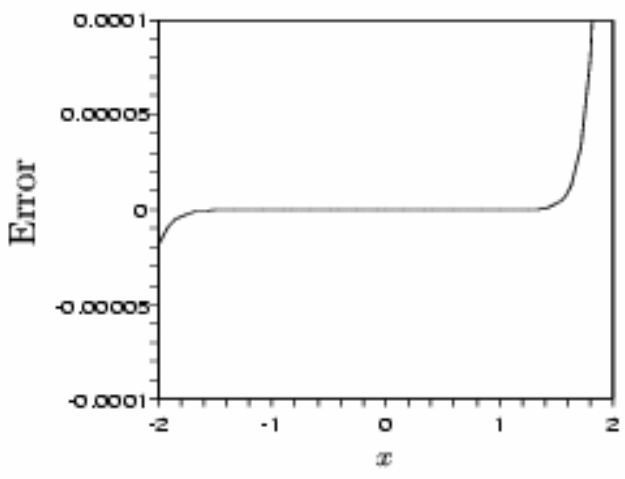

(a)

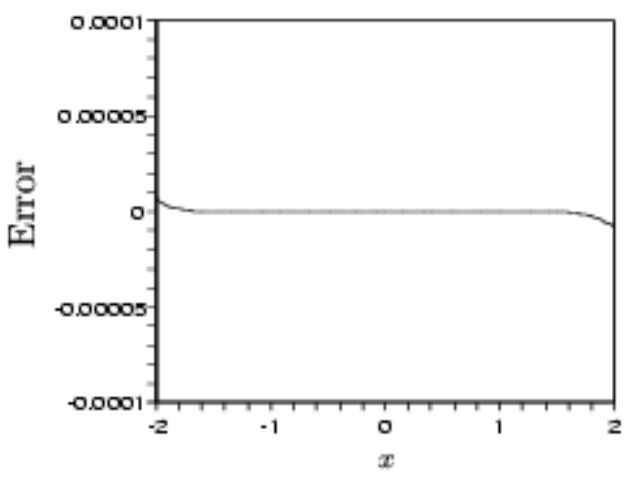

(b)

Fig. 2: (a): The error of VIM with sixth iteration for $u(x)$ (b): The error of VIM with sixth iteration for $v(x)$.

Example 3.2: [22] Consider the following nonlinear system of two integro-differential equations

$$
\begin{aligned}
& u^{\prime}(x)=1-\frac{1}{2} v^{\prime 2}(x)+\int_{0}^{x}((x-t) v(t)+u(t) v(t)) \mathrm{d} t, \\
& v^{\prime}(x)=2 x+\int_{0}^{x}\left((x-t) u(t)-v^{2}(t)+u^{2}(t)\right) \mathrm{d} t, \\
& u(0)=0, \quad v(0)=0,
\end{aligned}
$$


with the exact solutions

$$
u(x)=\sinh (x), \quad \mathrm{v}(\mathrm{x})=\cosh (\mathrm{x})
$$

According to (2) we have the following iteration formulations

$$
\begin{aligned}
& u_{k+1}(x)=u_{k}(x)-\int_{0}^{\mathrm{x}}\left[u_{k}^{\prime}(s)-1+\frac{1}{2} v_{k}^{\prime 2}(s)-\int_{0}^{\mathrm{s}}\left((s-t) v_{k}(t)+u_{k}(t) v_{k}(t)\right) \mathrm{d} t\right] \mathrm{d} s, \\
& v_{k+1}(x)=v_{k}(x)-\int_{0}^{\mathrm{x}}\left[v_{k}^{\prime}(s)-2 s-\int_{0}^{\mathrm{s}}\left((s-t) u_{k}(t)-v_{k}^{2}(t)+u_{k}^{2}(t)\right) \mathrm{d} t\right] \mathrm{d} s .
\end{aligned}
$$

With the initial approximation $u_{0}(x)=0$ and $v_{0}(x)=1$ which satisfy initial conditions, then we will have the below approximations:

$$
\begin{aligned}
& u_{1}(x)=x+\frac{1}{6} x^{3}, \\
& v_{1}(x)=1+\frac{1}{2} x^{2}, \\
& u_{2}(x)=x+\frac{1}{6} x^{3}+\frac{1}{24} x^{5}+\frac{1}{504} x^{7}, \\
& v_{2}(x)=1+\frac{1}{2} x^{2}+\frac{1}{24} x^{4}+\frac{1}{240} x^{6}+\frac{1}{2016} x^{8},
\end{aligned}
$$

It is obvious that the iterations converge to expand of exact solutions. Also, as the same of Example 1, obtained results by VIM and homotopy perturbation method on interval $[-1,1]$ are showed in Figures (3) and (4). We see again that variational iteration method is very effective and highly promising.

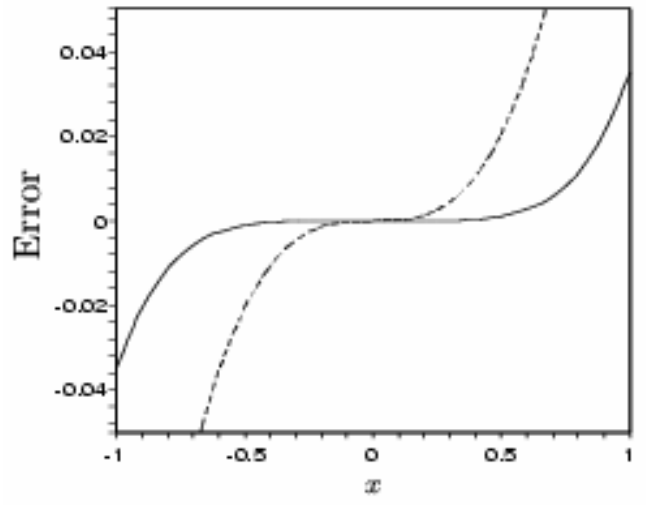

(a)

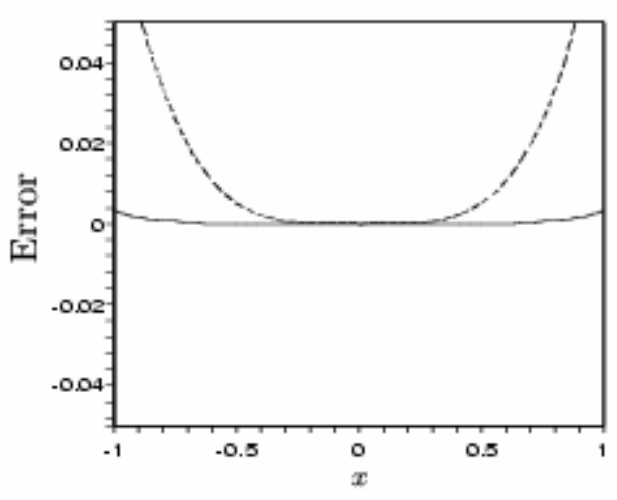

(b)

Fig. 3: (a): Solid line: The error of VIM with only second iteration for $u(x)$; dashed line: The error of homotopy method with 2 terms for $u(x)$; (b): Solid line: The error of VIM with second iteration for $v(x)$; dashed line: The error of homotopy method with 2 terms for $v(x)$. 


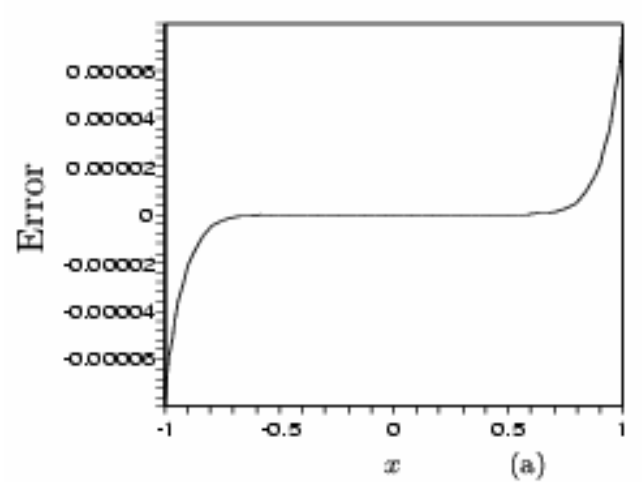

(a)

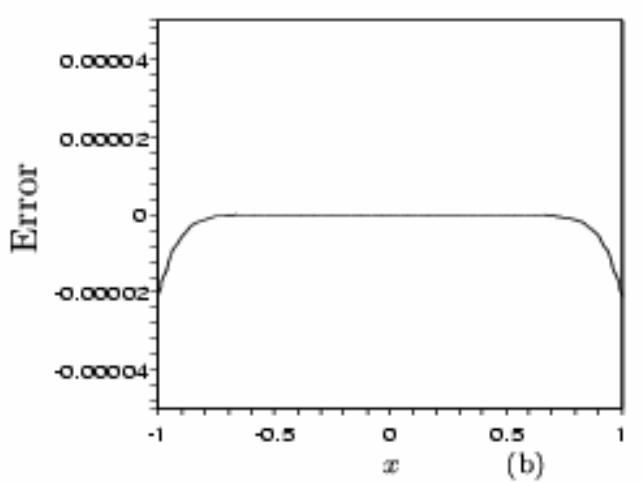

(b)

Fig. 4: (a): The error of VIM with fifth iteration for $u(x)$ (b): The error of VIM with fifth iteration for $v(x)$

Example 3.3: [22] Consider the following other nonlinear system of three integrodifferential equations

$$
\begin{aligned}
& u^{\prime \prime}(x)=x+2 x^{3}+2 v^{\prime 2}(x)-\int_{0}^{x}\left(v^{\prime 2}(t)+u(t) w^{\prime \prime}(t)\right) \mathrm{d} t, \\
& v^{\prime \prime}(x)=3 x^{2}-x u(x)+\int_{0}^{x}\left(t x v^{\prime}(t) u^{\prime \prime}(t)-w^{\prime}(t)\right) \mathrm{d} t, \\
& w^{\prime \prime}(x)=2-\frac{4}{3} x^{3}+u^{\prime \prime 2}(x)-2 u^{2}(x)+\int_{0}^{x}\left(x^{2} v(t)+u^{\prime 2}(t)+t^{3} w^{\prime \prime}(t)\right) \mathrm{d} t, \\
& u(0)=1, \quad u^{\prime}(0)=0, \quad v(0)=0, \quad v^{\prime}(0)=1, \quad w(0)=0, \quad w^{\prime}(0)=0,
\end{aligned}
$$

with the exact solutions

$$
u(x)=x^{2}, \quad v(x)=x, w(x)=3 x^{2} .
$$

Using the variational iteration method (2) with the initial approximations $u_{0}(x)=0$, $v_{0}(x)=1$ and $W_{0}(x)=0$ which satisfy initial conditions, gives

$$
\begin{gathered}
u_{k+1}(x)=u_{k}(x)+\int_{0}^{\mathrm{x}}(s-x)\left[u_{k}^{\prime \prime}(s)-s-2 s^{3}-2 v_{k}^{\prime 2}(s)+\int_{0}^{\mathrm{s}}\left(v_{k}^{\prime 2}(t)+u_{k}(t) w_{k}^{\prime \prime}(t)\right) \mathrm{d} t\right] \mathrm{d} s, \\
v_{k+1}(x)=v_{k}(x)+\int_{0}^{\mathrm{x}}(s-x)\left[v_{k}^{\prime \prime}(s)-3 s^{2}+s u_{k}(s)-\int_{0}^{\mathrm{s}}\left(t s v_{k}^{\prime}(t) u_{k}^{\prime \prime}(t)-w_{k}^{\prime}(t)\right) \mathrm{d} t\right] \mathrm{d} s, \\
w_{k+1}(x)=w_{k}(x)+\int_{0}^{\mathrm{x}}(s-x)\left[w_{k}^{\prime \prime}(s)-2+\frac{4}{3} s^{3}-u_{k}^{\prime 2}(s)+2 u_{k}^{2}(s)\right. \\
\left.-\int_{0}^{\mathrm{s}}\left(s^{2} v_{k}(t)+u_{k}^{\prime 2}(t)+t^{3} w_{k}^{\prime \prime}(t)\right) \mathrm{d} t\right] \mathrm{d} s .
\end{gathered}
$$

Then, we have the following primary approximations 


$$
\begin{aligned}
& u_{1}(x)=x^{2}+\frac{1}{10} x^{5}, \\
& v_{1}(x)=x+\frac{1}{4} x^{4}, \\
& w_{1}(x)=x^{2}-\frac{1}{15} x^{5}+\frac{1}{60} x^{6}, \\
& u_{2}(x)=x^{2}+\frac{4}{15} x^{5}-\frac{1}{60} x^{6}+\frac{197}{5040} x^{8}-\frac{1}{336} x^{9}+\frac{1}{7425} x^{11}-\frac{1}{26400} x^{12}, \\
& v_{2}(x)=x+\frac{1}{6} x^{4}+\frac{1}{630} x^{7}+\frac{41}{3360} x^{8}+\frac{1}{440} x^{11}, \\
& w_{2}(x)=3 x^{2}+\frac{2}{5} x^{5}-\frac{1}{30} x^{6}+\frac{13}{168} x^{8}-\frac{227}{30240} x^{9}+\frac{1}{1440} x^{10}+\frac{1}{3960} x^{11}-\frac{1}{6600} x^{12} .
\end{aligned}
$$

In order to show the efficiency and high accuracy of the present method we showed results on interval $[-1,1]$ graphically in Figure (5).

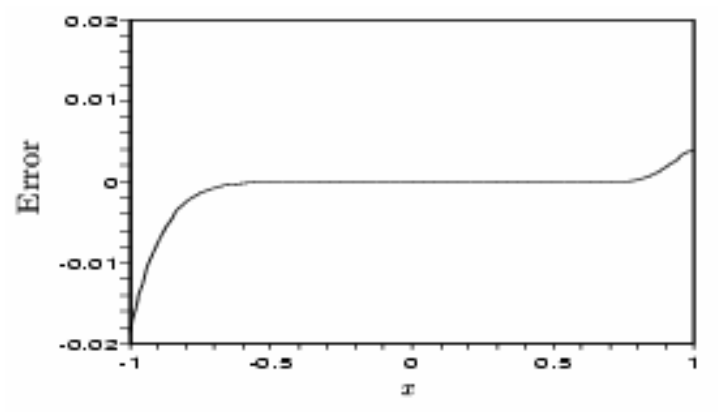

(a)

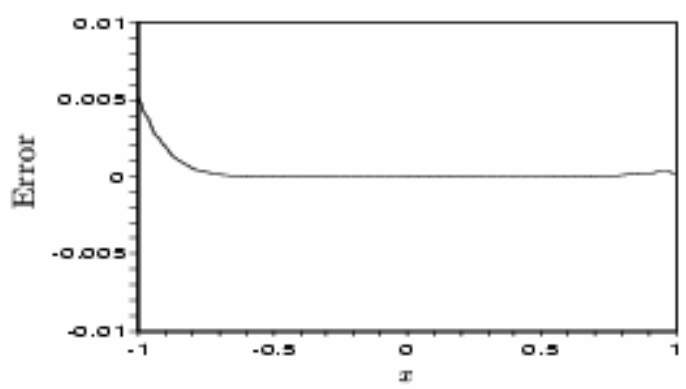

(b)

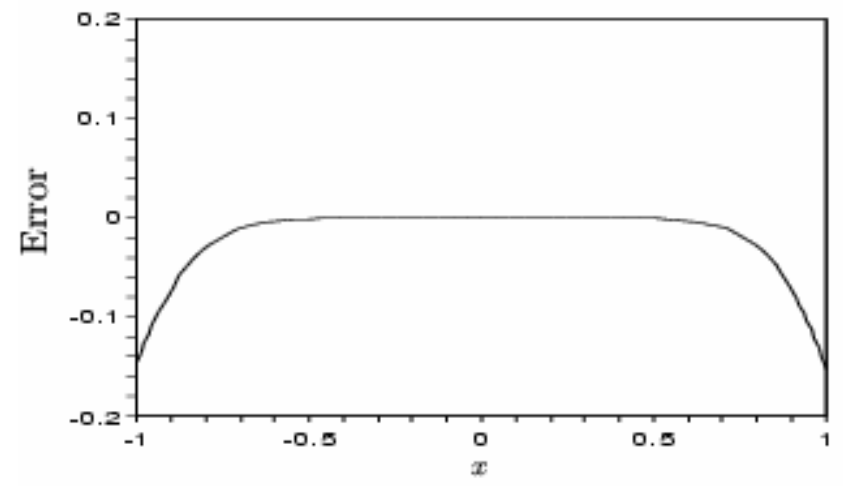

(c)

Fig. 5: (a): The error of VIM with sixth iteration for $u(x)$ (b): The error of VIM with sixth iteration for $v(x)$ (c): The error of VIM with sixth iteration for $w(x)$

Example 3.4: [26, 27] Now, let's test the VIM on the following linear system of two Volterra's integro-differential equations 


$$
\begin{aligned}
& u^{\prime}(x)=1+x+x^{2}-v(x)-\int_{0}^{x}(u(t)+v(t)) \mathrm{d} t, \\
& v^{\prime}(x)=-1-x+u(x)-\int_{0}^{x}(u(t)-v(t)) \mathrm{d} t, \\
& u(0)=1, \quad v(0)=-1,
\end{aligned}
$$

with the exact solutions

$$
u(x)=x+e^{x}, \quad v(x)=x-e^{x}
$$

Above system was solved in [26] and [27] that results are the same. Using the variational iteration method (2) with the initial approximations $u_{0}(x)=x+1$ and $v_{0}(x)=-1$, which satisfy initial conditions, gives

$$
\begin{aligned}
& u_{k+1}(x)=u_{k}(x)-\int_{0}^{\mathrm{x}}\left[u_{k}^{\prime}(s)-1-s-s^{2}+v_{k}(s)+\int_{0}^{\mathrm{s}}\left(u_{k}(t)+v_{k}(t)\right) \mathrm{d} t\right] \mathrm{d} s, \\
& v_{k+1}(x)=v_{k}(x)-\int_{0}^{\mathrm{x}}\left[v_{k}^{\prime}(s)+1+s-u_{k}(s)+\int_{0}^{\mathrm{s}}\left(u_{k}(t)-v_{k}(t)\right) \mathrm{d} t\right] \mathrm{d} s .
\end{aligned}
$$

Then, we have the following primary approximations

$$
\begin{array}{ll}
u_{1}(x)=1+2 x+\frac{1}{2} x^{2}+\frac{1}{6} x^{3}, & v_{1}(x)=-1-x^{2}-\frac{1}{6} x^{3} \\
u_{2}(x)=1+2 x+\frac{1}{2} x^{2}+\frac{1}{3} x^{3}+\frac{1}{12} x^{4}, & v_{2}(x)=-1-\frac{1}{2} x^{2}-\frac{1}{6} x^{3}-\frac{1}{12} x^{4}-\frac{1}{60} x^{5} .
\end{array}
$$

It is obvious that the iterations converge to expand of exact solutions. In order to show the efficiency and high accuracy of the present method we compared results on interval $[-2,2]$ with homotopy perturbation method and Adomian decomposition method graphically in Figure (6). Also, the obtained solutions with 10 iterations are showed in Figure (7) graphically.

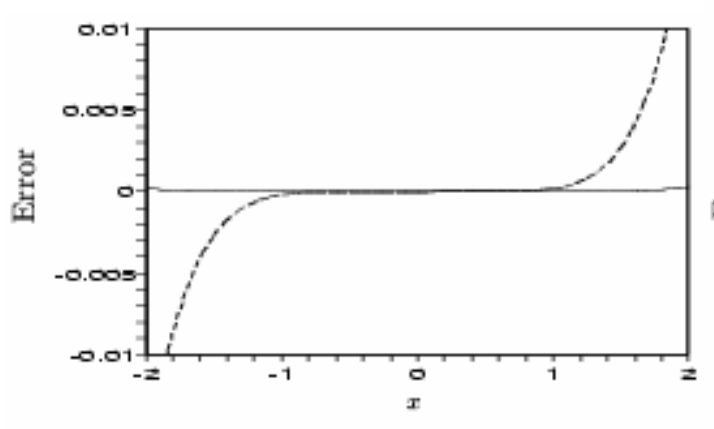

(a)

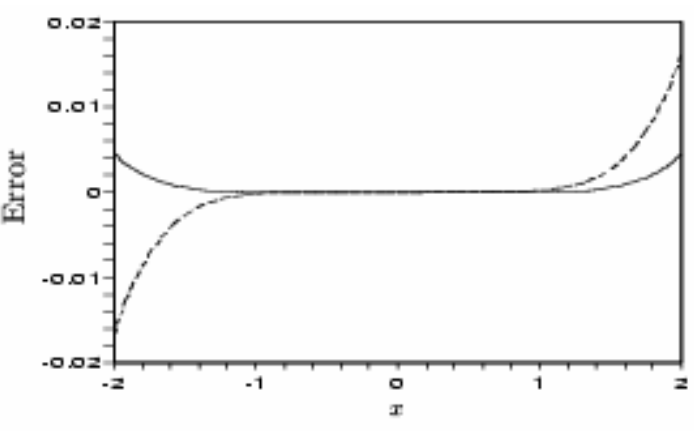

(b)

Fig. 6: (a): Solid line: The error of VIM with seventh iteration for $u(x)$; dashed line: The error of homotopy method with 7 terms for $u(x)$; (b): Solid line: The error of VIM with seventh iteration for $v(x)$; dashed line: The error of homotopy method with 7 terms for $v(x)$. 


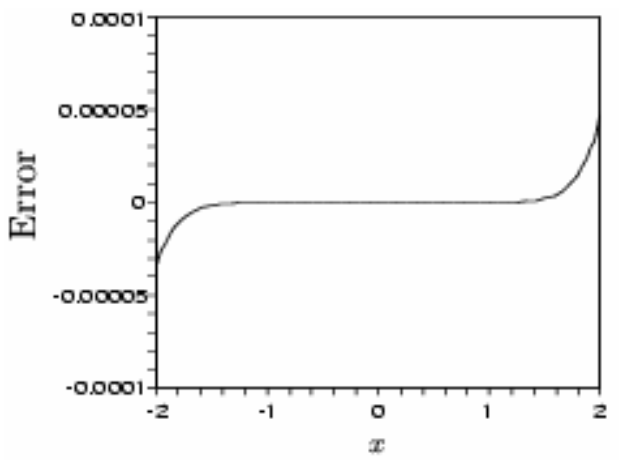

(a)

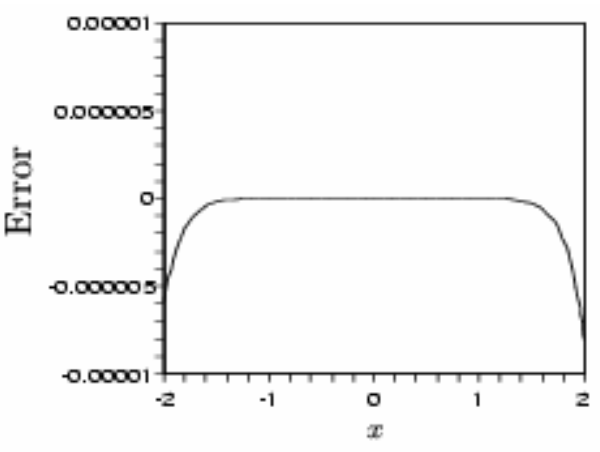

(b)

Fig. 7: (a): The error of VIM with tenth iteration for $u(x)$ (b): The error of VIM with tenth iteration for $v(x)$

\section{CONCLUSIONS}

In this paper, we have studied system of nonlinear integro-differential equations with the variational iteration method. The initial approximation was selected arbitrary not in form of the exact solution with unknown constants. The results showed that the variational iteration method is remarkably effective and performing is very easy. In addition, it has more accuracy than homotopy perturbation method and Adomian decomposition method for this kind of problems.

\section{REFERENCES}

1. J. H. He, A new approach to nonlinear partial differential equations, Comm. Nonlinear Sci. Numer. Simul. 2 (4) (1997) 230-235.

2. J. H. He, A variational approach to nonlinear problems and its application, Mech. Applic. 20 (1) (1998) 30-31.

3. M. Inokuti et al. General use of the Lagrange multiplier in nonlinear mathematical physics, in: S. Nemat-Nassed, ed., Variational method in the Mechanics of Solids (Pergamon Press, 1978) 156-162.

4. J. H. He, Variational iteration method for autonomous ordinary differential systems, Appl. Math. Comput. 118 (2-3) (2000) 115-123.

5. J. H. He, Variational principle for some nonlinear partial differential equations with variable coefficients, Chaos, Solitons I\& Fractals 19 (2004) 847-851.

6. M. A. Abdou, A.A. Soliman, New applications of variational iteration method, Physica D 211 (1-2) (2005) 1-8.

7. M. A. Abdou, A.A. Soliman, Variational iteration method for solving Burger's and coupled Burger's equations, J. Comput. Appl. Math. 181 (2005) 245-251.

8. S. Momani, S. Abuasad, Application of He's variational iteration method to Helmholtz equation, Chaos, Solitons \&\& Fractals 27 (2006) 1119-1123.

9. Z. M. Odibat, S. Momani, Application of variational iteration method to nonlinear differential equations of fractional order, Int. J. Nonlinear Sci. Numer. Simul. 7 (2006) 27-36. 
10. J. H. He, Some asymptotic methods for strongly nonlinear equations, Int. J. Modern Phys. B 20 (10) (2006) 1141-1199.

11. E. M. Abulwafa, M.A. Abdou, A.A. Mahmoud, The solution of nonlinear coagulation problem with mass loss, Chaos Solitons I\& Fractals 26 (2006) 313-330.

12. N. Bildik, A. Konuralp, The use of variational iteration method, differential transform method and Adomian decomposition method for solving different types of nonlinear partial differential equations, Int. J. Nonlinear Sci. Numer. Simul. 7 (1) (2006) $65-70$.

13. H. Wang, H. M. Fu, H. F. Zhang, et al., A practical thermodynamic method to calculate the best glass-forming composition for bulk metallic glasses, Int. J. Nonlinear Sci. Numer. Simul. 8 (2) (2007) 171-178.

14. L. Xu, J. H. He, Y. Liu, Electrospun nanoporous spheres with Chinese drug, Int. J. Nonlinear Sci. Numer. Simul. 8 (2) (2007) 199-202.

15. F. Z. Sun, M. Gao, S .H. Lei, et al., The fractal dimension of the fractal model of dropwise condensation and its experimental study, Int. J. Nonlinear Sci. Numer. Simul. 8 (2) (2007) 211-222.

16. T. L. Bo, L. Xie, X. J. Zheng, Numerical approach to wind ripple in desert, Int. J. Nonlinear Sci. Numer. Simul. 8 (2) (2007) 223-228.

17. M. El-Shahed, Application of He's homotopy perturbation method to Volterra's integro-differential equation, Int. J. Nonlinear Sci. Numer. Simul. 6 (2) (2005) 163-168.

18. S. M. El-Sayed, D. Kaya, S. Zarea, The decomposition method applied to solve high-order linear Volterra-Fredholm integro-differential equations, Int. J. Nonlinear Sci. Numer. Simul. 5 (2) (2004) 105-112.

19. S. Q. Wang, J. H. He, Variational iteration method for solving integro-differential equations, Phys. Lett. A 367 (2007) 188-191.

20. M. Ghasemi, M. Tavassoli Kajani, E. Babolian, Application of He's homotopy perturbation method to nonlinear integro-differential equations, Appl. Math. Comput. 188 (2007) 538-548.

21. J, Saberi-Nadjafi, M. Tamamgar, The variational iteration method: A highly promising method for solving the system of integro-differential equations, Comput. Math. Appl., (Article in press).

22. J. Biazar, H. Ghazvini, M. Eslami, He's homotopy perturbation method for systems of integro-differential equations, Chaos, Solitons I\& Fractals (Article in press).

23. J. H. He, Variational iteration method-a kind of non-linear analytical technique: some examples Int. J. Nonlinear Mech. 34 (1999) 699-708.

24. J. H. He and X. H. Wu, Construction of solitary solution and compacton-like solution by variational iteration method, Chaos, Solitons I\& Fractals 29 (2006) 108113.

25. J. H. He, The variational iteration method for eighth-order initial-boundary value problems, Phys. Scr. 76 (2007) 680-682.

26. J. Biazar, Solution of systems of integral-differential equations by Adomian decomposition method, Appl. Math. Comput. 168 (2005) 1232-1238.

27. E. Yusufolv\{g\}lu, An efficient algorithm for solving integro-differential equations system, Appl. Math. and Comput. 192 (2007) 51-55. 
28. M. Dehghan, M. Tatari, Identifying an unknown function in a parabolic equation with overspecified data via He's variational iteration method, Chaos, Solitons and Fractals, Volume 36, (2008), 157-166.

29. M. Dehghan, F. Shakeri, Application of He's variational iteration method for solving the Cauchy reaction-diffusion problem, Journal of Computational and Applied Mathematics, Volume 214, (2008), 435-446.

30. M. Dehghan, F. Shakeri, Numerical solution of a biological population model using He's variational iteration method, Computers and Mathematics with Applications, 54 (2007) 1197-1209.

31. M. Dehghan, F. Shakeri, Approximate solution of a differential equation arising in astrophysics using the variational iteration method, New Astronomy, 13 (2008) 53-59. 